\title{
Macrophage-specific Mycobacterium tuberculosis genes: identification by green fluorescent protein and kanamycin resistance selection
}

\author{
Correspondence \\ B. S. Srivastava \\ drbrahm@gmail.com \\ C. Locht \\ camille.locht@pasteur-lille.fr
}

Received 26 July 2006

Revised 7 November 2006

Accepted 9 November 2006

\author{
Vikas Srivastava, ${ }^{1}$ Carine Rouanet, ${ }^{2}$ Ranjana Srivastava, ${ }^{1}$ B. Ramalingam, ${ }^{2}$ \\ Camille Locht $^{2}$ and Brahm S. Srivastava ${ }^{1}$ \\ ${ }^{1}$ Microbiology Division, Central Drug Research Institute, Lucknow, India \\ ${ }^{2}$ INSERM U629, Institut Pasteur de Lille, 1 rue du Professor Calmette, F-59019 Lille Cedex, \\ France
}

\begin{abstract}
Mycobacterium tuberculosis survives and multiplies inside macrophages of its host by modulating the expression of several genes essential for in vivo survival. An in vivo expression system has been developed, based on green fluorescent protein and kanamycin resistance, to identify $M$. tuberculosis genes which appear to be up-regulated in infected macrophages. A promoter-trap shuttle vector, pLL192, was constructed, containing a streptomycin resistance gene as selection marker and an artificial bicistronic operon composed of the promoterless green fluorescent protein $(g f p)$ gene, followed by the kanamycin resistance gene. A unique BamHI site upstream of the gfp gene allowed for insertion of promoter libraries. The vector was validated by the use of known regulated or constitutive $M$. tuberculosis promoters. In addition, an $M$. tuberculosis genomic DNA library was inserted into pLL192 and then introduced into Mycobacterium bovis BCG. The recombinant BCG cells were then used to infect the J774A.1 murine macrophage-like cell line in the presence of kanamycin. Several recombinant BCG cells were thereby selected that were resistant to kanamycin within infected macrophages, but were sensitive to kanamycin when grown in vitro. The kanamycin resistance phenotype was paralleled by the fluorescence phenotype. After nucleotide sequencing, the corresponding genes were identified as mce1A, PE_PGRS63(RV3097c), Rv2232, Rv1026, Rv1635c, viuB, Rv2231(cobC) and Rv0997.

Real-time PCR analysis using RNA isolated at various time points from $M$. tuberculosis and $M$. bovis BCG grown in vitro and within macrophages, confirmed the up-regulation of these genes. The level of up-regulation varied from 2- to 40-fold in macrophages compared to growth in vitro.
\end{abstract}

\section{INTRODUCTION}

Tuberculosis is the second leading cause of mortality by infectious diseases in the human population, killing about 1.8 million people worldwide [www.who.int/mediacentre/ factsheets/fs104/en/print.html (accessed on 6 November 2004)]. The problem has worsened with the spread of AIDS, which may lead to reactivation of dormant tubercle bacilli and make patients more prone to infection by nontuberculous mycobacteria.

Tuberculosis is treated by a combination of four drugs which act on different targets. In recent years, Mycobacterium tuberculosis isolates resistant to one or more of these drugs have been spreading, which seriously hampers

Abbreviation: IVET, in vivo expression technology. successful control measures (WHO, 1997). Novel drugs acting on new targets are therefore urgently needed.

There are several important macromolecules in the mycobacterial cell which are vital for survival of the organism in host cells and progression of the disease (Smith, 2003). These macromolecules may either be constitutively expressed or their expression may be upregulated within the host. Finding such targets is the key to developing new drugs.

M. tuberculosis infects by aerosol and is ingested by alveolar macrophages. Contrary to most other invading microorganisms, which are killed within the macrophages, the tubercle bacillus survives and grows in the unfavourable conditions inside the macrophages. It has been reported that alterations in gene expression in the mycobacteria and in the macrophages take place, which allow the mycobacteria to 
survive (Barker et al., 1998; Dubnau et al., 2002; Hobson et al., 2002). Studies with other pathogens have shown that genes which are up-regulated during infection are often important for pathogenicity (Chiang et al., 1999). Therefore, the identification of mycobacterial genes that are upregulated in infected macrophages may provide targets for drug design or vaccine development.

Different approaches have been used to identify microbial genes that play a role in the interaction with host cells and thus in virulence (Jacobs et al., 1991). These include signature-tagged transposon mutagenesis (Camacho et al., 1999), in vivo-induced antigen technology (Deb et al., 2002), substractive hybridization (Plum \& Clark-Curtiss, 1994), selective capture of transcribed sequences (Graham \& ClarkCurtiss, 1999) and in vivo expression technology (IVET; Mahan et al., 1993). Several important genes have been identified in mycobacteria, including hspX (Yuan et al., 1998), and genes encoding isocitrate lyase (McKinney et al., 2000), mycobacterial cell entry protein (Arruda et al., 1993) and glutamine synthetase (Harth \& Horwitz, 1999).

Compared to other techniques, IVET has the advantage of identifying bacterial genes in the host environment. It was originally developed to identify virulence genes of Salmonella typhimurium (Mahan et al., 1993) and this elegant approach is being applied now to other pathogenic bacteria. In the present investigation, we have used a modified IVET strategy based on a promoter-trap plasmid vector. The plasmid contains two reporter genes, $g f p$, encoding green fluorescent protein, and kan, encoding kanamycin resistance, in a transcriptional fusion downstream of a promoter cloning site. An M. tuberculosis promoter library was screened in macrophages on the basis of $g f p$ expression and kanamycin resistance. Expression levels of genes belonging to the identified promoters were then individually confirmed by real-time PCR analysis.

\section{METHODS}

Bacterial strains and growth conditions. Escherichia coli $\mathrm{DH} 5 \alpha$ $\left[\mathrm{F}^{-}\right.$( $880 d \Delta$ lacZ M15) recA1 endAl gyrA96 thi-1 hsdR17 $\left(\mathrm{r}_{\mathrm{K}}^{-} \mathrm{m}_{\mathrm{K}}^{+}\right)$ supE44 relA1 deoR $\triangle$ (lacIZYA-argF)U169; Invitrogen] and NM522 $\left[\mathrm{F}^{\prime}\right.$ proAB lacl ${ }^{\mathrm{q}} \Delta$ lacZ $M 15 /$ supE thi $\Delta($ lac-proAB $) \Delta($ hsdMS-mcrB $) 5$ $\left(\mathrm{r}_{\mathrm{K}}^{-} \mathrm{m}_{\mathrm{K}}^{+}\right) \mathrm{McrB}^{-}$; MBI Fermentas] were used for cloning of the different constructs and the library. Mycobacterium tuberculosis H37Rv, Mycobacterium smegmatis $\mathrm{mc}^{2} 155$, Mycobacterium bovis BCG Pasteur strain (isolate 1173P2; WHO) were employed in this study. E. coli was grown in Luria-Bertani (LB) broth or plated on LB solidified with $1.5 \%(w / v)$ agar. Mycobacteria were grown in liquid Sauton medium (Sauton, 1912), Middlebrook 7H9 medium (Difco) supplemented with ADC $(0.2 \%$ glucose, $0.5 \%$ bovine serum albumin fraction V, $0.0003 \%$ beef catalase) or solid Middlebrook 7H10/7H11 agar (Difco) + OADC enrichment (Becton Dickinson). All cultures were grown at $37^{\circ} \mathrm{C}$ with or without shaking. Antibiotics were added into the media when required at the following concentrations: streptomycin, 10-25 $\mu \mathrm{g} \mathrm{ml}^{-1}$; kanamycin, 5-25 $\mu \mathrm{g} \mathrm{ml}^{-1}$; cycloheximide, $50 \mu \mathrm{g} \mathrm{ml}^{-1}$; gentamicin, $50 \mu \mathrm{g} \mathrm{ml}^{-1}$.

Fluorescence measurement. Fluorescence was measured by using a FACS Calibur (Becton Dickinson) and the Cell quest software

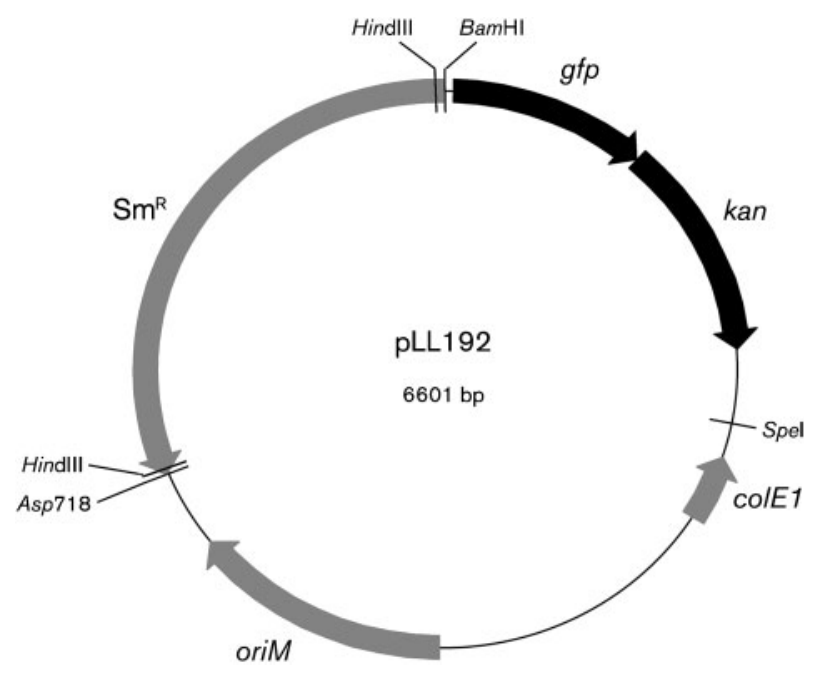

Fig. 1. Plasmid map of pLL192.

provided by the supplier. The bacterial population was gated by using side and forward scatter parameters and then analysed for fluorescence. The results are expressed as the percentage population fluorescing and the mean fluorescence intensity.

Construction of promoter-trap vector pLL192. The E. coliMycobacterium shuttle vector pMV261 (Stover et al., 1991), containing an $E$. coli ( $\operatorname{col} E)$ and a mycobacterial (oriM) origin of replication, as well as a kanamycin resistance gene and the mycobacterial hsp60 promoter, was chosen to construct pLL192, as shown in Fig. 1. The gene encoding streptomycin resistance $\left(\mathrm{Sm}^{\mathrm{r}}\right)$ was amplified by PCR

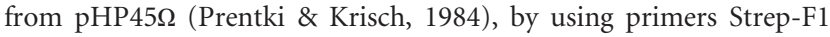
(5'-AATGGGGTACCGGTGATTGATTGAGC-3') and Strep-R1 (5'CCGAAACGCGCGAGGCAAGCTTA- $\left.3^{\prime}\right)$. The amplified fragment was directly inserted into the PCRII-TOPO vector (TOPO TA cloning; Invitrogen). The $\mathrm{Sm}^{\mathrm{r}}$ gene to be used as a selection marker was then recovered by a BamHI/Asp718 restriction and inserted into pMV261 previously digested with the same enzymes. After transformation of E. coli NM522, $\mathrm{Sm}^{\mathrm{r}}$ clones were isolated. The promoterless $g f p$ gene was amplified by PCR from pJFX2 (Triccas et al., 1999) by using primers GFP Forw2 (5'-GCGGATCCTCTAGATTTAAGAAG$\left.3^{\prime}\right)$ and GFPBlunt (5'-TAAGATATCATTTGTATAGTTCATCCATGCC-3') containing a Bam $\overline{\mathrm{HI}}$ and an EcoRV site (underlined), respectively. The promoterless kan gene was amplified by PCR from pMV261 by using the primers KanR1 (5'-GGGTCTGACGCTCAGTGGAA-3') and KanBlunt (5'-GGTGTGTTGACCCATATTCAACGGGAA- $3^{\prime}$ ), the latter containing a HincII site (underlined). The two amplified fragments were digested respectively with BamHI/EcoRV and HincII/SpeI and inserted in a single step into the BamHI/SpeIdigested $\mathrm{Sm}^{\mathrm{r}}$ pMV261 derivative. The GFPBlunt and KanBlunt primers were defined such that, after cloning, the start codon of the $k a n$ gene overlaps the stop codon of the $g f p$ gene. The final construct, named pLL192, contains an artificial bicistronic operon composed of the promoterless $g f p$ gene, followed by the kan gene. The presence of a unique BamHI site immediately upstream of this transcriptional fusion allows for the insertion of promoter libraries.

Construction of the M. tuberculosis promoter-trap library. $M$. tuberculosis $\mathrm{H} 37 \mathrm{Rv}$ genomic DNA was isolated as described previously by Marmur (1961) and Baulard et al. (1996), and partially digested with Sau3AI. After agarose gel electrophoresis, fragments of 100-800 bp were purified (QIAquick Gel Extraction Kit; Qiagen) and inserted into dephosphorylated BamHI-digested pLL192. After 
transformation of E. coli DH5 $\alpha$, DNA was extracted from approximately 100000 recombinant colonies and electroporated into $M$. bovis BCG (Gene Pulser II; Bio-Rad; $2.5 \mathrm{kV}, 25 \mu \mathrm{F}, 800 \Omega$ ). The mycobacteria were then plated onto Middlebrook 7H10 agar containing streptomycin and cycloheximide. More than $10000 \mathrm{Sm}^{\mathrm{r}}$ clones with inserts were obtained. Realizing fully that this number may not represent the whole genome, these clones were harvested and pooled in Middlebrook 7H9 medium (Difco) supplemented with $10 \%$ (v/v) ADC. Glycerol (15\%, v/v) was added and the mycobacteria were stored at $-80^{\circ} \mathrm{C}$. Before use, the recombinant mycobacterial pool was grown in Sauton medium to mid-exponential phase.

Macrophage infection. The J774A.1 mouse macrophage cell line was grown in RPMI (Gibco-BRL) or Dulbecco's modified Eagle medium (DMEM; Gibco-BRL) supplemented with $10 \%$ heatinactivated fetal calf serum (FCS; Gibco-BRL), at $37^{\circ} \mathrm{C}$ in a $5 \%$ $\mathrm{CO}_{2}$ humidified atmosphere. The cells were seeded in 24-well cell culture clusters (Corning) $\left(5 \times 10^{5}\right.$ cells per well in a $1 \mathrm{ml}$ volume) and allowed to adhere overnight. The macrophages were then infected with a mycobacterial suspension at an m.o.i. of 1:5. After $4 \mathrm{~h}$ of infection, macrophage monolayers were washed several times to remove the remaining extracellular bacteria and the cells were incubated for an additional $24-48 \mathrm{~h}$ in fresh medium containing gentamicin $\left(50 \mu \mathrm{g} \mathrm{ml}^{-1}\right)$ to kill extracellular bacteria. The macrophages were then lysed with PBS/ $0.05 \%$ SDS. The fluorescence of intracellular bacteria was analysed by flow cytometry and compared to that observed for bacteria incubated under the same conditions but in the absence of macrophages. In the case of the M. tuberculosis promoter library, macrophages were infected with recombinant BCG for $4 \mathrm{~h}$ as described above, several washes were performed, and then they were incubated in fresh culture medium with gentamicin and kanamycin $\left(5 \mu \mathrm{g} \mathrm{ml}^{-1}\right)$ for an additional $24-48 \mathrm{~h}$ to select specifically recombinant BCG containing a promoter activated ex vivo, therefore conferring resistance against this antibiotic. A kanamycin concentration of $5 \mu \mathrm{g} \mathrm{ml}^{-1}$ was selected because more than $80 \%$ killing of BCG harbouring empty pLL192 was caused by $1 \mu \mathrm{g}$ kanamycin $\mathrm{ml}^{-1}$ in the medium. Macrophages were then lysed with PBS plus $0.05 \%$ SDS. The recovered bacteria were plated onto Middlebrook 7H10 agar plates supplemented with streptomycin and cycloheximide. The growing colonies were then checked individually for their kanamycin resistance in vitro.

RNA isolation and real-time PCR. For RNA isolation, J774A.1 macrophages were infected with $M$. tuberculosis or M. bovis BCG at an m.o.i. of $1: 5$. After $4 \mathrm{~h}$ of infection, the macrophages were washed twice with DMEM medium without FCS and then incubated in DMEM medium containing FCS and gentamicin $\left(50 \mu \mathrm{g} \mathrm{ml}^{-1}\right)$. At different time points $(24,36$ and $48 \mathrm{~h}$ ), the cells were harvested, washed with DMEM medium without FCS and then lysed with Tween normal saline containing $0.1 \%$ Tween $(\mathrm{w} / \mathrm{v})$ and $0.1 \%(\mathrm{w} / \mathrm{v})$ SDS. The debris of lysed macrophages was removed by centrifugation at $200 \mathrm{~g}$. The supernatant containing bacteria and lysate of macrophages was mixed with two volumes of RNAprotect provided in the Qiagen RNAeasy kit. Bacteria were then pelleted by centrifugation at 10000 r.p.m. $(9000 \mathrm{~g})$ for $7 \mathrm{~min}$ and the supernatant was discarded. The bacterial pellet was resuspended in TE + lysozyme $\left(10 \mathrm{mg} \mathrm{ml}^{-1}\right)$ and briefly sonicated after adding RLT buffer from the RNAeasy kit. The RNA was then isolated by using the RNAeasy kit and quantified spectrophotometrically. About 5-10 $\mu$ g RNA was obtained from each macrophage experiment. Using the same protocol, RNA was isolated from in vitro-grown cultures of M. tuberculosis and $M$. bovis BCG by resuspending the bacteria in TE + lysozyme, sonication and extraction by using the RNAeasy kit. RNA was also extracted from bacteria incubated in DMEM medium containing FCS, to eliminate the effect of serum components and DMEM, and no difference was observed (data not shown). For real-time PCR analysis, each RNA sample was treated with Promega DNase and

Table 1. Primers used for promoter and real-time PCR

\begin{tabular}{|c|c|}
\hline Gene/amplicon & Primer sequences $\left(5^{\prime}-3^{\prime}\right)$ \\
\hline \multirow[t]{2}{*}{ Acetamidase gene upstream } & CGGGATCCGAAGTGACGCGGTCTCAAGCGTC \\
\hline & CGGGATCCAACTACCTCGGGCATGTGGACTC \\
\hline \multirow[t]{2}{*}{ Rv0467/icl gene upstream } & GAAGATCTCTGGCTGAACCCGCGCTGG \\
\hline & GAAGATCTAGACAACTCCTTAACGGTCTTTC \\
\hline \multirow[t]{2}{*}{$16 \mathrm{~S}$ rRNA } & TCCCGGGCCTTGTACACA \\
\hline & CCACTGGCTTCGGGTGTTA \\
\hline \multirow[t]{2}{*}{ Rv2232 } & TTCCGACACGCGCTCAACCAC \\
\hline & CAACCGCGGGCGCTGTAGTC \\
\hline \multirow[t]{2}{*}{ Rv1026 } & GGTCGGGCTGGCTGGAACG \\
\hline & GGGCACCCGCGAAAGATGAAT \\
\hline \multirow[t]{2}{*}{ Rv3097c/PE_PGRS63 } & GCGATCGCGGCTTTGTTTTC \\
\hline & CTCGGCGGCGGCATACC \\
\hline \multirow[t]{2}{*}{ Rv0169/mce1A } & ACGGTGTTCGGCGGTAAGTATGTG \\
\hline & CGCGCTCAGGGTCAGGTTC \\
\hline \multirow[t]{2}{*}{ Rv2895c/viuB } & GACCTGCCCACCGCGAAG \\
\hline & ACGGACCGGCTACCCC \\
\hline \multirow[t]{2}{*}{ Rv0997 } & GGCCGGCATAGCTGGTGTGGAT \\
\hline & GGGTCTTGCGGTCGGGTTCG \\
\hline \multirow[t]{2}{*}{ Rv1635c } & CGCCGGCGTGGTGGTTTTT \\
\hline & CCGCCGCTGGGTGTTGC \\
\hline \multirow[t]{2}{*}{ Rv2231/cobC } & GAGCGCTGCCGGAATCACG \\
\hline & CTCGCCGGGAACCCAATCAGC \\
\hline
\end{tabular}




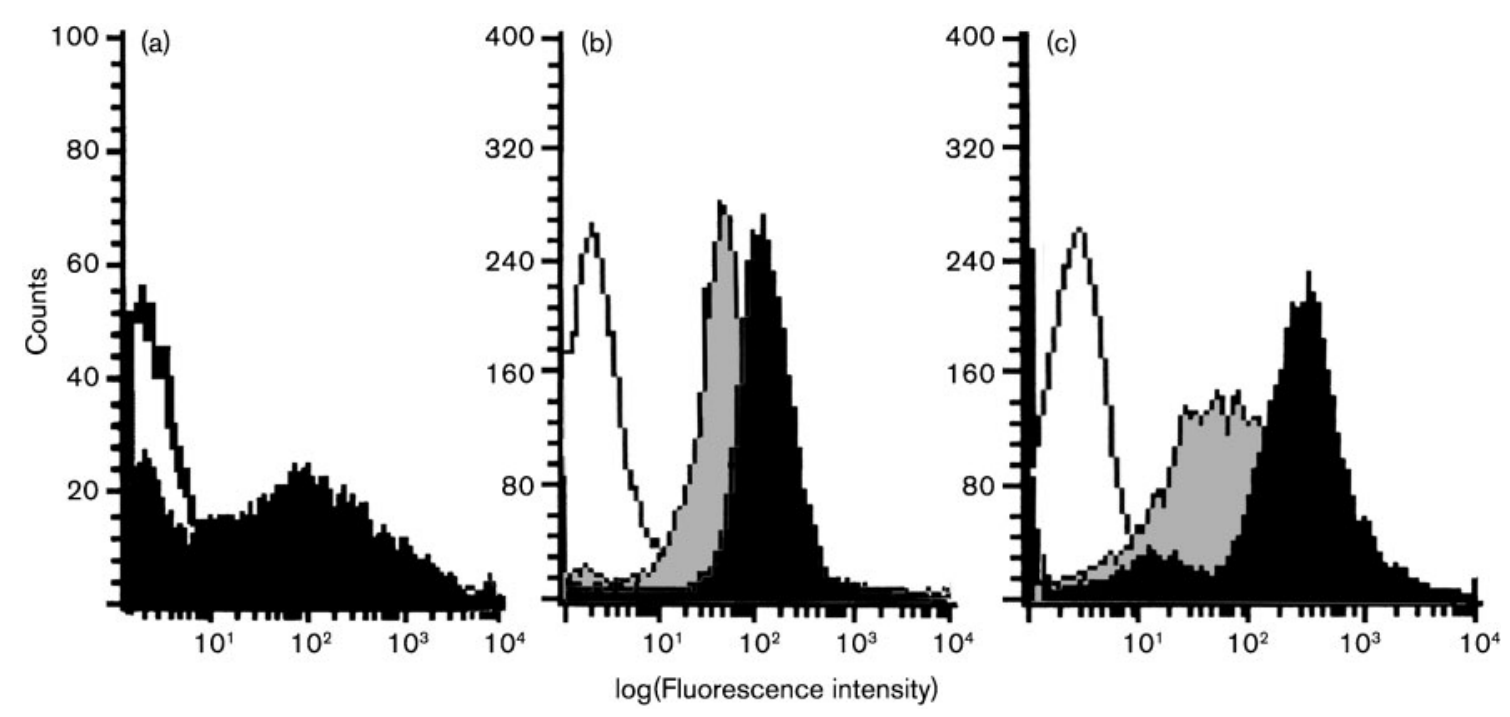

Fig. 2. GFP fluorescence of recombinant BCG harbouring pLL192 alone and with various promoters cloned into the $B a m H I$ site. (a) In vitro fluorescence of vector alone (white area) and with hsp60 promoter (black area). (b) In vitro fluorescence of vector alone (white area) and with the icl promoter in the presence of glucose- (grey area) and acetate-containing broth (black area). (c) In vitro fluorescence of vector alone (white area) and with the acetamidase promoter in the presence of 0.5 (grey area) and $2 \mathrm{mg}$ acetamide $\mathrm{ml}^{-1}$ (black area) in the broth.

heat-inactivated according to the manufacturer's instructions. About 200-400 ng RNA was used per reaction. In each set of reactions, $16 S$ rRNA was used for normalization. Each reaction was repeated three times with three independent RNA samples in a Cepheid SmartCycler II. Primers used in real-time PCR are listed in Table 1. A control without reverse transcriptase was included in each set of reactions, and a DNA melting point curve analysis was also done for each sample. Relative expression levels were determined using the $2^{-\Delta \Delta C_{\mathrm{T}}}$ method of Livak \& Schmittgen (2001), assuming that the PCR efficiencies were the same as seen from the slope of the reaction curves. The results were expressed as -fold induction of expression compared to the in vitro control.

\section{RESULTS}

\section{Construction of vector pLL192 and its validation with known promoters}

To identify mycobacterial promoters specifically expressed under defined conditions, we constructed pLL192 (Fig. 1). This E. coli-Mycobacterium shuttle vector contains two promoterless reporter genes, one encoding a mutated form of the green fluorescent protein $(g f p)$ and the second corresponding to the kanamycin resistance gene ( $k a n)$. These two genes are organized into a single transcription unit located immediately downstream from a unique BamHI site, allowing for the cloning of promoter libraries. To validate pLL192 as a promoter-trap vector, the $g f p:: k a n$ fusion was placed under the control of three different mycobacterial promoters, namely the constitutive $h s p 60$ promoter (Stover et al., 1991), the icl (isocitrate lyase) promoter (McKinney et al., 2000), known to be induced under specific physiological conditions, and the acetamidase gene promoter (Payton et al., 1999), whose induction is acetamide-dependent. The resulting plasmids were electroporated into $M$. smegmatis $\mathrm{mc}^{2} 155$ and M. bovis BCG and analysed for fluorescence and kanamycin sensitivity. Empty pLL192 was used as a negative control. Fluorescence was observed with bacteria harbouring the $h s p 60$ promoter in pLL192 (Fig. 2a) and these bacteria were resistant to up to $25 \mu \mathrm{g}$ kanamycin $\mathrm{ml}^{-1}$, compared to the negative control for which more than $80 \%$ killing was observed at $1 \mu \mathrm{g}$ kanamycin $\mathrm{ml}^{-1}$. When the strain containing the pLL192 derivative with the $i c l$ promoter was grown in Middlebrook 7H9 medium containing $10 \mathrm{mM}$ acetate or glucose, fluorescence intensity was found to depend on the presence or absence of acetate or glucose (Fig. 2b). Finally, the construct containing the acetamidase gene promoter showed fluorescence intensities proportional to the concentrations of acetamide used in the media (0.2-2.0 mg ml${ }^{-1}$; Fig. 2c).

\section{Macrophage infections and isolation of ex vivo-induced promoters}

A library of Sau3AI-digested M. tuberculosis chromosomal DNA was generated using pLL192. The library was then introduced into M. bovis BCG and the recombinant BCG strains, along with the control BCG containing pLL192 without insert, were used to infect macrophages.

Kanamycin $\left(5 \mu \mathrm{g} \mathrm{ml}^{-1}\right)$ was found to kill effectively the intracellular BCG control containing pLL192 without insert. This correlated well with the lack of fluorescence (data not shown). Macrophages infected with BCG control containing pLL192 without insert were grown in the presence or absence of kanamycin. After incubation, the macrophages were lysed and the bacteria were plated onto Middlebrook $7 \mathrm{H} 10$ 


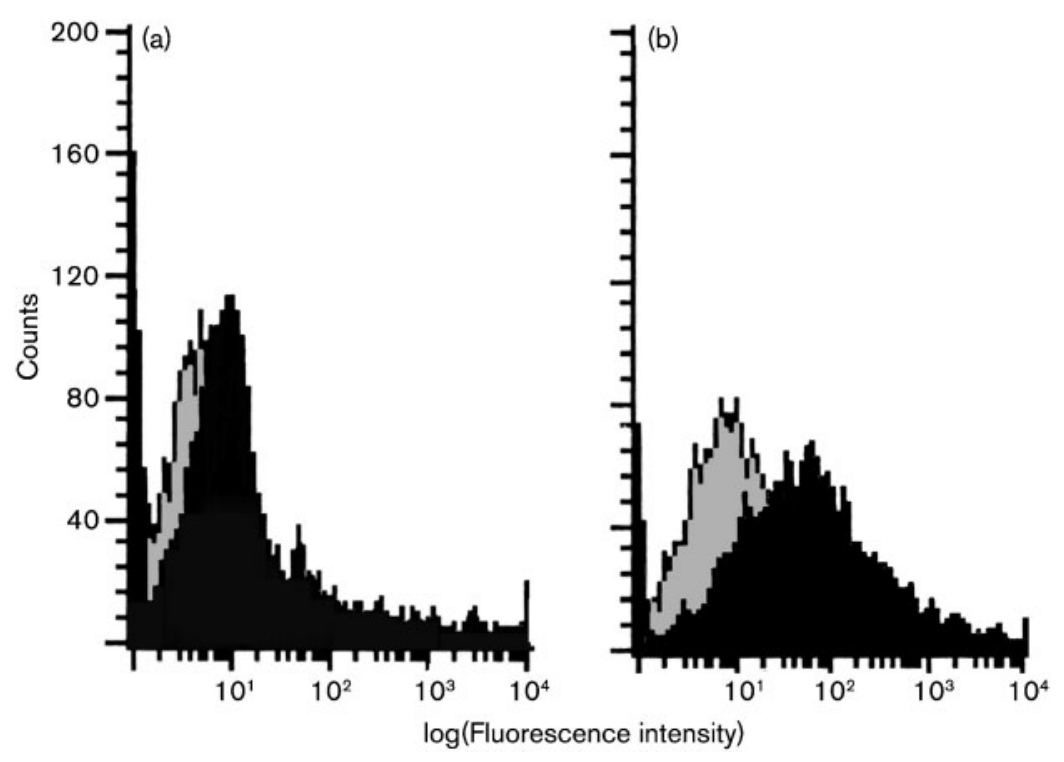

Fig. 3. GFP fluorescence of (a) vector alone (grey area) and clone Rv2232 (black area) in vitro and (b) vector alone (grey area) and clone Rv2232 (black area) within macrophages after $24 \mathrm{~h}$ of infection. agar with or without kanamycin. Approximately $1.5 \times 10^{4}$ colonies grew on the plates when the bacteria came from macrophages grown in the absence of kanamycin and plated onto Middlebrook 7H10 agar without kanamycin. However, no colonies were observed for the control BCG after growth in macrophages cultured in the presence of kanamycin.

When BCG cells containing the promoter library were used to infect macrophages, grown in the presence of kanamycin and subsequently plated onto Middlebrook 7H10 agar without kanamycin, 14 colonies were detected. Eight of these failed to grow on Middlebrook 7H10 agar in the presence of kanamycin. These eight clones were then used again to infect macrophages grown with or without kanamycin. No difference in the survival of the BCG clones was observed between these two conditions, confirming that these clones could survive in macrophages in the presence of kanamycin. However, they remained sensitive to kanamycin when grown in vitro on Middlebrook 7H10 agar, suggesting up-regulation of the corresponding promoters in macrophages ex vivo. When analysed for fluorescence, all eight clones were found to fluoresce strongly ex vivo, but showed no or weak fluorescence in vitro, as shown for one example in Fig. 3.

Plasmid DNA from each of the eight clones was isolated and the inserts at the BamHI site were sequenced. The sequences thus obtained were then compared to the genome sequence of $M$. tuberculosis (Cole et al., 1998) to identify the corresponding genes, and they are listed in Table 2. Real-time PCR analysis using RNA isolated at various time points from $M$. tuberculosis and M. bovis BCG grown in vitro and within macrophages was then carried out to confirm the up-regulation of these genes. The results are shown in Fig. 4(a, b). After normalization to the $16 \mathrm{~S}$ rRNA, 2- to 40-fold induction of the specific genes was observed both in M. tuberculosis and M. bovis BCG in infected macrophages, compared to in vitro growth. However, some

Table 2. Genes identified by IVET selection

\begin{tabular}{|ll|}
\hline Gene & \multicolumn{1}{c|}{ Function of product } \\
\hline Rv2232 & Conserved hypothetical protein \\
Rv1026 & $\begin{array}{c}\text { Conserved hypothetical protein; could be involved in adaptive phase of } \\
\text { bacterial response to amino acid starvation }\end{array}$ \\
Rv3097c/PE_PGRS63 & PE-PGRS family protein; probable triacyl glycerol lipase (esterase/lipase) \\
Rv0169/mce1A & Involved in host-cell invasion (entry and survival in macrophages) \\
Rv2231/cobC & Possible aminotransferase involved in cobalamin biosynthesis \\
Rv1635c & Conserved transmembrane protein \\
Rv0997 & Hypothetical protein \\
Rv2895c/viuB & Homologue of vibriobactin utilization protein (ViuB) of Vibrio cholerae, \\
& possibly involved in mycobactin utilization \\
\end{tabular}




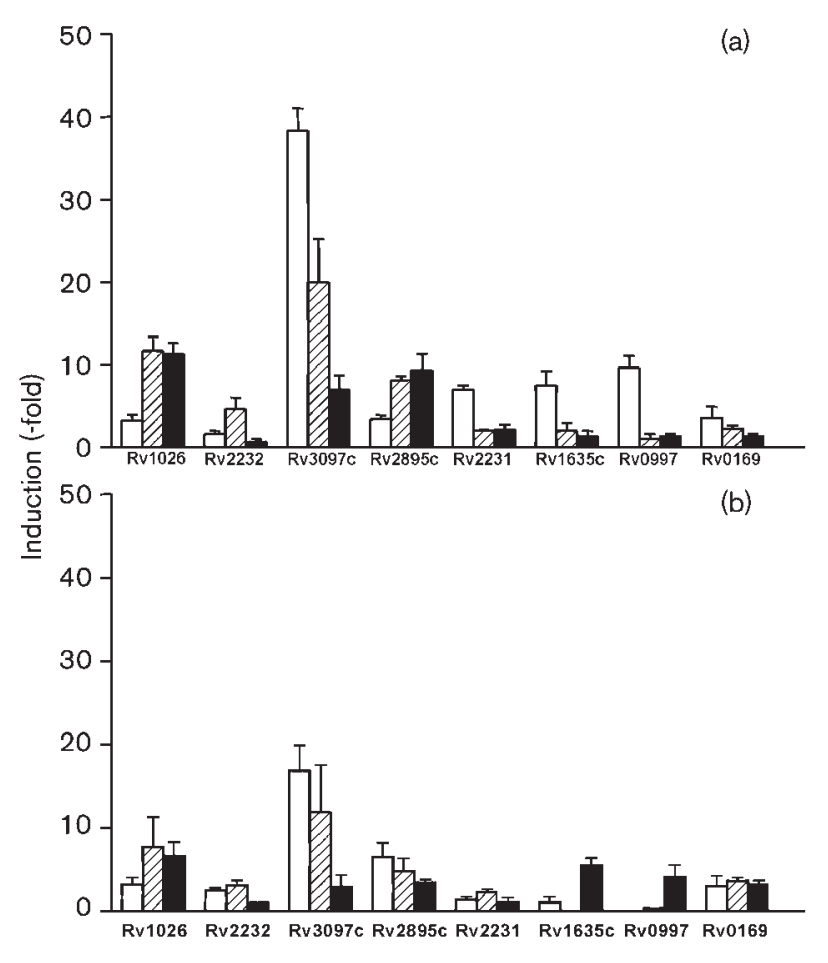

Fig. 4. Real-time measurement of up-regulation, compared to the in vitro sample of genes, within macrophages in (a) $M$. tuberculosis H37Rv and (b) $M$. bovis BCG at various time points post-infection: 24 (white bars), 36 (hatched bars) and $48 \mathrm{~h}$ (black bars).

difference in gene expression was observed between $M$. tuberculosis and M. bovis BCG.

All the genes showed up-regulation at varying levels, and it varied at different time points. PE_PGRS63 (Rv3097c) showed the strongest induction in both the strains, but decreased at $48 \mathrm{~h}$ post-infection.

\section{DISCUSSION}

The identification of $M$. tuberculosis genes expressed intracellularly within macrophages may contribute significantly to the development of new strategies to control tuberculosis. In this investigation we have used a new promoter probe vector with two reporter genes in transcriptional fusion, independent of added inducers, and constructed an $M$. tuberculosis library as a source for the selection of promoter fragments responding to various environmental stimuli. GFP displays natural fluorescence and kanamycin is known to be effective against intracellular bacteria (Kremer et al., 1995; Maurin \& Raoult, 2001). The vector was validated by using different known constitutive and inducible promoters. The combination of GFP and selection by kanamycin allowed us to select $M$. tuberculosis promoters which were activated and up-regulated within macrophages compared to mycobacteria grown in vitro. Vectors combining GFP with the counterselectable SacB protein (Triccas et al., 1999), or kanamycin pre-selection with SacB post-selection (Saviola et al., 2003), were employed to select M. tuberculosis genes expressed within the macrophages. The simultaneous expression of fluorescence and kanamycin resistance phenotypes conferred by pLL192 in the presence of an activated promoter makes the selection process easy in a single step and has the advantage of usability in vivo. In the first round, eight clones with the desired phenotype were identified. The activities of the reporter genes under the control of these promoters were minimal in vitro as judged by weak or absent fluorescence and kanamycin sensitivity on 7H10 agar, but were high within macrophages. This was confirmed and quantified by RT-PCR. In all cases, expression was up-regulated several-fold within macrophages. Expression of PE_PGRS63 was higher in macrophages than during in vitro culture. Within the macrophages, its expression was maximal after $24 \mathrm{~h}$ (the first time point) and then decreased. The expression of mce1A was observed within $24 \mathrm{~h}$ of infection and was similar in both $M$. tuberculosis and $M$. bovis BCG. The presence of mcelA in many species of mycobacteria, including mycobacteria other than tuberculosis-causing species, suggests that it is an important gene for entry and survival (Arruda et al., 1993).

Rv1026 is a conserved hypothetical protein whose exact function is not yet known, but it may be involved in an adaptive response to amino acid starvation and has been classified as an essential gene by Himarl-based transposon mutagenesis of M. tuberculosis (Sassetti et al., 2003). Hence, up-regulation of Rv1026 might reflect the adaptive response to stress within the macrophage.

Acquisition of iron is an essential feature of many bacterial pathogens as iron is required for growth and survival. Mycobacteria have evolved various mechanisms to acquire iron (De Voss et al., 1999), but the role of the potential mycobactin utilization protein ViuB encoded by Rv2895c has not been studied. However, it shows homology to the vibriobactin utilization protein (ViuB) of Vibrio cholerae (Butterton \& Calderwood, 1994), and may thus be involved in intracellular removal of iron from iron-mycobactin complexes.

Rv2232 is a putative phosphatase whose function remains to be defined. The expression of Rv2232 has been shown to be up-regulated significantly in SCID mice, but not in BALB/C mice (Talaat et al., 2004), suggesting that this gene has a role in immunocompromised hosts. Rv2231c (CobC) is a putative transferase involved in cobalamin biosynthesis. Using Himarl-based transposon mutagenesis in M. tuberculosis H37Rv, it was demonstrated that Rv2231c corresponds to an essential gene in H37Rv (Sassetti et al., 2003). The role of Rv1635c and Rv0997 is not known.

The deduced functions of selected genes are consistent with conditions encountered within the macrophages. The induction of their expression observed ex vivo may therefore represent a strategy employed by $M$. tuberculosis to promote 
the infection and to enhance intracellular survival. This investigation has led to the selection of promoters that are activated over $48 \mathrm{~h}$ after the beginning of the infection. Thus it might have facilitated identification of promoters which are induced early and then down-regulated, which is supported by the RT-PCR data. However, the survival of the bacilli and their multiplication inside macrophages might also involve other genes displaying an up-regulated expression during extended periods of time. Therefore, it might be fruitful to subject the promoter library to selection at different time points, up to 6-7 days, within the macrophages. We appreciate that the present investigation is not a comprehensive study because the promoter library may not be complete as random restriction by Sau3AI has most certainly led to the loss of some promoter fragments involved in intracellular growth. Fragmenting chromosomal DNA by other means may thus be helpful to construct more representative libraries to be used in the context of this vector for the identification of promoters up- or downregulated under specific environmental conditions.

\section{ACKNOWLEDGEMENTS}

The work reported in this paper has been supported by a project jointly awarded to Brahm S. Srivastava and Camille Locht by the Indo-French Centre for Promotion of Advanced Research, New Delhi. The Indian investigators are thankful to the Director of the Institute for laboratory facilities and Mr A. L. Vishwakarma for excellent technical support with flow cytometry. V.S. gratefully acknowledges the Junior and Senior Research Fellowships of the Indian CSIR. This is communication no. 6917 of the CDRI.

\section{REFERENCES}

Arruda, S., Bomfim, G., Knights, R., Huima-Byron, T. \& Riley, L. W. (1993). Cloning of an Mycobacterium tuberculosis DNA fragment associated with entry and survival inside cells. Science 261, 1454-1457.

Barker, L. P., Brooks, D. M. \& Small, P. L. C. (1998). The identification of Mycobacterium marinum genes differentially expressed in macrophage phagosomes using promoter fusions to green fluorescent protein. Mol Microbiol 29, 1167-1177.

Baulard, A., Kremer, L. \& Locht, C. (1996). Efficient homologous recombination in fast-growing and slow-growing mycobacteria. J Bacteriol 178, 3091-3098.

Butterton, J. R. \& Calderwood, S. B. (1994). Identification, cloning, and sequencing of a gene required for ferric vibriobactin utilization by Vibrio cholerae. J Bacteriol 176, 5631-5638.

Camacho, L. R., Ensergueix, D., Perez, E., Gicquel, B. \& Guilhot, C. (1999). Identification of a virulence gene cluster of Mycobacterium tuberculosis by signature-tagged transposon mutagenesis. Mol Microbiol 34, 257-267.

Chiang, S. L., Mekalanos, J. J. \& Holden, D. W. (1999). In vivo genetic analysis of bacterial virulence. Annu Rev Microbiol 53, 129-154.

Cole, S. T., Brosch, R., Parkhill, J., Garnier, T., Churcher, C., Harris, D., Gordon, S. V., Eiglmeier, K., Gas, S. \& other authors (1998). Deciphering the biology of Mycobacterium tuberculosis from the complete genome sequence. Nature 393, 537-544.
Deb, D. K., Dahiya, P., Srivastava, K. K., Srivastava, R. \& Srivastava, B. S. (2002). Selective identification of new therapeutic targets of Mycobacterium tuberculosis by IVIAT approach. Tuberculosis (Edinb) 82, 175-182.

De Voss, J. J., Rutter, K., Schroeder, B. G. \& Barry, C. E., III (1999). Iron acquisition and metabolism by mycobacteria. J Bacteriol 181, 4443-4451.

Dubnau, E., Fontan, P., Manganelli, R., Soares-Appel, S. \& Smith, I. (2002). Mycobacterium tuberculosis genes induced during infection of human macrophages. Infect Immun 70, 2787-2795.

Graham, J. E. \& Clark-Curtiss, J. E. (1999). Identification of Mycobacterium tuberculosis RNAs synthesized in response to phagocytosis by human macrophages by selective capture of transcribed sequences (SCOTS). Proc Natl Acad Sci U S A 96, 11554-11559.

Harth, G. \& Horwitz, M. A. (1999). An inhibitor of exported Mycobacterium tuberculosis glutamine synthetase selectively blocks the growth of pathogenic mycobacteria in axenic culture and in human monocytes: extracellular proteins as potential novel drug targets. J Exp Med 189, 1425-1436.

Hobson, R. J., McBride, A. J., Kempsell, K. E. \& Dale, J. W. (2002). Use of an arrayed promoter-probe library for the identification of macrophage-regulated genes in Mycobacterium tuberculosis. Microbiology 148, 1571-1579.

Jacobs, W. R., Jr, Kalpana, G. V., Cirillo, J. D., Pascopella, L., Snapper, S. B., Udani, R. A., Jones, W., Barletta, R. G. \& Bloom, B. R. (1991). Genetic systems for mycobacteria. Methods Enzymol 204, 537-555.

Kremer, L., Baulard, A., Estaquier, J., Poulain-Gotefroy, O. \& Locht, C. (1995). Green fluorescent protein as a new expression marker in mycobacteria. Mol Microbiol 17, 913-922.

Livak, K. J. \& Schmittgen, T. D. (2001). Analysis of relative gene expression data using real-time quantitative PCR and the $2^{-\Delta \Delta C_{\mathrm{T}}}$ Method. Methods 25, 402-408.

Mahan, M. J., Slauch, J. M. \& Mekalanos, J. J. (1993). Selection of bacterial virulence genes that are specifically induced in host tissues. Science 259, 686-688.

Marmur, J. A. (1961). Procedure for the isolation of deoxyribonucleic acid from microorganisms. J Mol Biol 3, 208-218.

Maurin, M. \& Raoult, D. (2001). Use of aminoglycosides in treatment of infections due to intracellular bacteria. Antimicrob Agents Chemother 45, 2977-2986.

McKinney, J. D., Honer zu Bentrup, K., Munoz-Elias, E. J., Miczak, A., Chen, B., Chan, W. T., Swenson, D., Sacchettini, J. C., Jacobs, W. R., Jr \& Russell, D. G. (2000). Persistence of Mycobacterium tuberculosis in macrophages and mice requires the glyoxylate shunt enzyme isocitrate lyase. Nature 406, 735-738.

Payton, M., Auty, R., Delgoda, R., Everett, M. \& Sim, E. (1999). Cloning and characterization of arylamine $\mathrm{N}$-acetyltransferase genes from Mycobacterium smegmatis and Mycobacterium tuberculosis: increased expression results in isoniazid resistance. J Bacteriol 181, 1343-1347.

Plum, G. \& Clark-Curtiss, J. E. (1994). Induction of Mycobacterium avium gene expression following phagocytosis by human macrophages. Infect Immun 62, 476-483.

Prentki, P. \& Krisch, H. M. (1984). In vitro insertional mutagenesis with a selectable DNA fragment. Gene 29, 303-313.

Sassetti, C. M., Boyd, D. H. \& Rubin, E. J. (2003). Genes required for mycobacterial growth defined by high density mutagenesis. Mol Microbiol 48, 77-84.

Sauton, B. (1912). Sur la nutrition minérale du bacilli tuberculeux. C R Acad Sci III Sci Vie 155, 860-863.

Saviola, B., Woolwine, S. C. \& Bishai, W. R. (2003). Isolation of acid-inducuble genes of Mycobacterium tuberculosis with the use of 
recombinase-based in vivo expression technology. Infect Immun 71, $1379-1388$.

Smith, I. (2003). Mycobacterium tuberculosis pathogenesis and molecular determinants of virulence. Clin Microbiol Rev 16, 463-496.

Stover, C. K., de la Cruz, V. F., Fuerst, T. R., Burlein, J. E., Benson, L. A., Bennett, L. T., Bansal, G. P., Young, J. F., Lee, M. H. \& other authors (1991). New use of BCG for recombinant vaccines. Nature 351, 456-460.

Talaat, A. M., Lyons, R., Howard, S. T. \& Johnston, S. A. (2004). The temporal expression profile of Mycobacterium tuberculosis infection in mice. Proc Natl Acad Sci U S A 101, 4602-4607.

Triccas, J. A., Berthet, F. X., Pelicic, V. \& Gicquel, B. (1999). Use of fluorescence induction and sucrose counterselection to identify
Mycobacterium tuberculosis genes expressed within host cells. Microbiology 145, 2923-2930.

WHO (1997). Anti-tuberculosis drug resistance in the world. The WHO/IUATLD Global Project on Anti-tuberculosis Drug Resistance Surveillance 1994-1997 (WHO/TB/97.229). Geneva: World Health Organization.

Yuan, Y., Crane, D. D., Simpson, R. M., Zhu, Y. Q., Hickey, M. J., Sherman, D. R. \& Barry, C. E., III (1998). The 16-kDa alpha-crystallin (Acr) protein of Mycobacterium tuberculosis is required for growth in macrophages. Proc Natl Acad Sci U S A 95, 9578-9583.

Edited by: W. Bitter 